\title{
An Investigation into the Use of Logical and Rhetorical Tactics within Eristic Argumentation on the Social Web
}

\author{
Tom Blount \\ Web and Internet Science \\ ECS \\ University of Southampton \\ tb12g09@ecs.soton.ac.uk
}

\author{
David E. Millard \\ Web and Internet Science \\ ECS \\ University of Southampton \\ dem@ecs.soton.ac.uk
}

\author{
Mark J. Weal \\ Web and Internet Science \\ ECS \\ University of Southampton \\ mjw@ecs.soton.ac.uk
}

\begin{abstract}
Argumentation is a key aspect of communications and can broadly be broken down into problem solving (dialectic) and quarrelling (eristic). Techniques used within argumentation can likewise be classified as fact-based (logical), or emotion/audience-based (rhetorical). Modelling arguments on the social web is a challenge for those studying computational argumentation as formal models of argumentation tend to assume a logical argument, whereas argumentation on the social web is often largely rhetorical. To investigate the application of logical versus rhetorical techniques on the social web, we bring together two ontologies used for modelling argumentation and online communities respectively, the Argument Interchange Format and the Semantic Interlinked Online Communities project. We augment these with our own ontology for modelling rhetorical argument, the Argumentation on the Social Web Ontology, and trial our additions by examining three case studies following argumentation on different categories of social media. Finally, we present examples of how rhetorical argumentation is used in the context of the social web and show that there are clear markers present that can allow for a rudimentary estimate for the classification of a social media post with regards to its contribution to a discussion.
\end{abstract}

\section{Categories and Subject Descriptors}

H.4.3 [Information Systems Applications]: Communications Applications-Social media

\section{Keywords}

argumentation; dialectic; eristic; logic; rhetoric; social web

\section{INTRODUCTION}

Argumentation is fundamental to human communication - it is how people share new information and new ideas, and propose courses of action that see them carried out $[5,12]$ and can be (broadly) separated into two categories based on the intended outcome: dialectic, in which the participants are engaged in rational discourse with the aim of either discovering the particular truth behind a matter, or formulating a solution to a problem [14], and eristic, in which

Permission to make digital or hard copies of all or part of this work for personal or classroom use is granted without fee provided that copies are not made or distributed for profit or commercial advantage and that copies bear this notice and the full citation on the first page. Copyrights for components of this work owned by others than ACM must be honored. Abstracting with credit is permitted. To copy otherwise, or republish, to post on servers or to redistribute to lists, requires prior specific permission and/or a fee. Request permissions from Permissions@acm.org.

HT '15 September 01 - 04, 2015, Guzelyurt, TRNC, Cyprus

(C) 2015 ACM. ISBN 978-1-4503-3395-5/15/09...\$15.00.

DOI: http://dx.doi.org/10.1145/2700171.2791052 the participants are quarrelling with the aim of being seen to win [7]. Orthogonally to this, there are the notions of logic and rhetoric. While often used in modern parlance as a pejorative term, rhetoric is the art of discourse and convincing an audience based on one's knowledge of the topic at hand and of the audience themselves. Logical argumentation uses the facts of a case to draw conclusions but, it is important to note that the "facts" do not necessarily need to be correct: they may be warped to fit a particular purpose, or even outright fabricated. The key element is that the argument relies on these facts and the reasoning between them, even if fallacious. In contrast, rhetorical argument focuses on swaying an audience to one's cause by other means, such as appealing to camaraderie or making threats. There is a tendency to view argumentation tactics in relatively stark terms: that dialectic/logical arguments are good and eristic/rhetorical arguments are bad, which leads to eristic and rhetorical argumentation being discounted from formal models. However, this should be resisted: logical argument can also be used in a hostile manner and, by contrast, eristic or rhetorical arguments are often used recreationally, for humour or catharsis [18].

As the social web grows, the potential for using it to investigate how truly massive communities interact, communicate and argue increases dramatically. However, the social web presents a number of challenges for extracting and analysing arguments, particularly due to use of informal language [17], and by the number of distinct "biomes" on the social web with their own constraints and cultures [8]. There are also challenges when considering maintaining the social web as an inclusive platform for diverse and vibrant discussion. Because of the tendency for users to interact with others who are similar in terms of traits and beliefs, sites can become "echo-chambers" in which well-known views and opinions are repeated, little original content is produced and there is virtually no dissent or debate $[4,19]$. Such spaces can quickly become stale or, at worst, incredibly hostile to those with opposing views, culminating at is most extreme in anti-social behaviour including vulgar abuse, threats of sexual violence, and death threats [20,6]. Disregarding these interactions from argumentation models is a mistake; indeed, accurately modelling them is the first step towards understanding how social media is used, and creating tools and environments that discourage these types of abuse to facilitate more social argumentation. As a result, current models must be combined and adapted to be fit for purpose when examining the social web.

\section{BACKGROUND}

\subsection{Argument Interchange Format}

The Argument Interchange Format (AIF) is a framework for representing argumentation as a directed graph [3]. Created as part of the Argument Web project [15], the AIF is primarily a description, 
with specifications in a number of languages including RDF and SQL. Data, claims and conclusions are modelled by Information nodes (I-nodes). I-nodes are linked by intermediary Scheme nodes (S-nodes). These S-nodes are subdivided into three applications: Rule of Inference Applications (RA-nodes), Conflict Applications (CA-nodes) and Preference Applications (PA-nodes). RA-nodes and CA-nodes denote an inference or conflict between one or more pieces of information, whereas PA-nodes denote a preference of one piece of information over another. In their work on an extension to the AIF, dubbed AIF+, Reed et al. build on the work of O'Keefe to differentiate between two separate notions of argumentation $[13,16]$ : the first, which they term argument $_{1}$, is a logically constructed set of claims and evidence used to back these claims (or attack other claims). The second, termed argument $_{2}$, refers to a dialogue - the exchange of ideas and opinions between two or more people. A result of this work was to introduce three new node types. Locutions (L-nodes) model locutionary acts in an argument 2 ; that is, they record precisely what was said. Transition Applications (TA-nodes) represent transitions between L-nodes, with associated forms such as a challenge or response. Illocutionary Applications (YA-nodes) represent the "illocutionary force" and serve to link each argument 1 to the overall argument 2 .

\subsection{Semantically Interlinked Online Communities}

The Semantically Interlinked Online Communities project (SIOC), a semantic-web vocabulary for representation social media, aims to enable the cross-platform, cross-service representation of data from the social web [2]. This allows for semantic representations of Sites, which hold Forums, which contain Posts, authored by a UserAccount (explicitly not a person, as a person can own and manage more than one UserAccount). While an extension to SIOC for the purposes of capturing and representing argumentation does exist [10], it is based on the Issue Based Information System (IBIS) model, a highly dialectic approach [9]. IBIS struggles to model eristic arguments due to the focus on the notion of issues and solutions, rather than quarrelling for its own sake.

\section{ARGUMENTATION ON THE SOCIAL WEB ONTOLOGY}

In our previous work, we examined the capability of existing frameworks used to capture and model both argumentation and social communities [1]. It became apparent that the AIF, while a powerful tool for modelling dialectic argument, lacked the ability to capture certain aspects of social argumentation. While some logical fallacies, such as the ad hominem attack can be suitably modelled within the AIF, the rhetorical force of simple abuse is difficult to capture. However, that does not mean it is not valuable to model such outbursts. A heckler in a debate, for example, may resort to throwing vulgarities, but by simply disrupting the proceedings they are voicing their dissent at the positions offered which can act to catalyse further argumentation on the subject between the main participants. While the AIF can model the locution, the rhetorical force behind it goes uncaptured. In addition, there are other socio-rhetorical tactics that are often employed, such as spamming to drown out other posters, deliberate deviation from the topic at hand, bringing up non-sequiturs in an attempt to derail the argument and "meta-argumentation" - criticising the way in which an opponent argues, but not the argument itself. There are also social features to consider: for example, the number of "Likes" or "Favourites" a post has can demonstrate audience support.
Key elements of the AIF and SIOC ontologies have been combined as parts of the Argumentation on the Social Web Ontology (ASWO), to explicitly capture the social component of argumentation on the social web. This is achieved by linking the concept of a Post with that of a Locution. We consider each post as an atomic unit of the dialogue, or argument ${ }_{2}$. In the majority of cases, a single locution will translate to a single self-contained argument ${ }_{1}$. In this paper we focus on extending ASWO to include rhetorical support and attack. While this is only one aspect of rhetorical argument they feature heavily in eristic dialogue and showcase both the positive and negative aspects of rhetorical argument. Rhetorical support is often relatively benign and can be used to show solidarity with other members of the dialogue or to encourage more dialectic debate. Consider the extract "I commend you for admitting that debt $\&$ deficits are important...If only more [people] felt the way you $d o$ ", which disagrees with the overall stance presented by their opponent, but commends them for conceding some common ground. Conversely, rhetorical attacks are often extremely hostile. They differ from logical attacks by attacking the person behind the argument rather than the argument itself (not to be confused with $\mathrm{ad}$ hominem, which attacks a person's argument by calling their character into question - these are logical, even though they are fallacious). We model the notion of rhetorical support and attack by introducing three new types of nodes to the ontology. Firstly, the Persona node represents a user's character and (purported) authority on a given subject, and is bound to a UserAccount. Introducing the notion of personas allows each UserAccount to present a different view of themselves (that can be supported or attacked accordingly) when engaging in multiple discussions or topics. PersonalConflict (PC-nodes) nodes link from a YA-node to a Persona node to denote this type of personal support and, likewise, PersonalSupport (PS-nodes) nodes follow the same structure to denote support of a person's intentions and character.

\section{INVESTIGATIONS}

To investigate the application of logical versus rhetorical techniques in eristic dialogue on the social web, and trial our augmentations made to the AIF and SIOC ontologies within the ASWO, we performed three case studies on arguments 2 taking place on different areas of the social web. A sample of two hundred and seventy posts from within three different threads were manually annotated using our modified framework, allowing us to analyse the relation between logical and rhetorical arguments 1 used, and compare the features of the annotation structure with the content of each post.

\subsection{Methodology}

A single topic of argumentation was chosen to be examined for three case studies, each representing a different social biome. To ensure the stimulation of debate, the selected topic needed to be controversial, have a large number of respondents and have been active for a long enough period of time to generate a rich and complete content. The Oct. 2013 United States government shutdown caused by Congress's failure to agree on a budget, and the following condemnation this received from the presidency, was a suitable match for these requirements. This topic was then tracked across three of Kaplan's social media categories: Twitter, a microblogging service; Facebook, a social network; and Reddit, a social news and networking site. The source of the posts themselves again needed to be both publicly available and have a large number of followers to ensure a maximally stimulated debate. As an authoritative public figure at the heart of the crisis, content from or relating to Barack Obama's social media profiles was chosen, and three posts dated 15 October 2013 were selected for study. 
Table 1: Metrics of discussions sampled from Twitter, Facebook and Reddit

\begin{tabular}{|l|c|c|c|c|}
\hline Metric & Twitter & Facebook & Reddit & Total \\
\hline Posts & 90 & 90 & 90 & 270 \\
\hline Direct replies & 77 & 0 & 67 & 144 \\
\hline Number of users & 26 & 85 & 43 & 154 \\
\hline Average posts per user & 3.5 & 1.1 & 2.1 & 1.8 \\
\hline Average words per post & 15.83 & 41.36 & 42.34 & 33.18 \\
\hline Average characters per post & 96.51 & 265.27 & 243.31 & 201.70 \\
\hline Time between first and last posts & 0d 6h 53m 40s & $3 \mathrm{~d} 4 \mathrm{~h} 51 \mathrm{~m} \mathrm{27s}$ & $3 \mathrm{~d} 0 \mathrm{~h} 50 \mathrm{~m} 12 \mathrm{~s}$ & $\mathrm{n} / \mathrm{a}$ \\
\hline Average time between posts & $04 \mathrm{~m} \mathrm{39s}$ & $51 \mathrm{~m} \mathrm{49s}$ & $49 \mathrm{~m} \mathrm{06s}$ & $35 \mathrm{~m} 11 \mathrm{~s}$ \\
\hline
\end{tabular}

Table 2: Summary of AIF and ASWO nodes in each sample

\begin{tabular}{|l|c|c|c|c|}
\hline Metric & Twitter & Facebook & Reddit & Total \\
\hline L-nodes & 90 & 90 & 90 & 270 \\
\hline TA-nodes & 52 & 9 & 15 & 76 \\
\hline YA-nodes & 58 & 74 & 70 & 202 \\
\hline I-nodes & 56 & 98 & 86 & 240 \\
\hline RA-nodes & 13 & 20 & 24 & 57 \\
\hline CA-nodes & 18 & 1 & 34 & 53 \\
\hline PA-nodes & 4 & 4 & 2 & 10 \\
\hline PS-nodes & 2 & 2 & 3 & 7 \\
\hline PC-nodes & 26 & 6 & 12 & 44 \\
\hline
\end{tabular}

Because of the volume of the data produced over the course of the tracked event and the time-intensive nature of manually annotating the data, it was necessary to sample the data to a more manageable size before annotation could take place. To ensure that the sampled graph maintained properties similar to those of the original graph, forest-fire sampling [11] was utilised to preserve the overall structure of the data. Table 1 shows an overview of the sample structures and some key characteristics of each thread. Manual annotation was required to derive the premises and conclusions (and subsequent relations) from each post. Each post is considered to contain zero or more separate arguments ${ }_{1}$. A YA-node is created for each argument ${ }_{1}$ made, and links the L-node to each I-node in the argument $_{1}$. Information that met one (or more) of the following criteria was not considered relevant: off-topic posts that do not relate to the topic being discussed (Example: "Ataturk did revolution! building moderate muslim network is oxymoron which has been destroy secular, democratic, rule of law in Turkey."); conversational posts (Example: "I thank you, have a good night!") and metaargumentation (Example: "Down voting = disagree Upvoting = agree" "The rules say explicitly not to do that..."). Repeated information does not create a new I-node; instead the YA-node links to the I-node already present. A TA-node is created to link two Locutions whenever a transition is present in the argument $_{2}-$ a step that contributes to the overall structure without providing any information, most often in the form of an interrogative. Support and attack between different I-nodes is denoted through the use of RA- and CA-nodes and preference with PA-nodes, while rhetorical support and attack utilises the new PS- and PC-nodes. Some nodes in the graph may not be complete as a result of the nature of sampling the graph. For example, it may be possible to detect that a user attacks another user's persona, but not exactly which user they are attacking. Table 2 shows an overview of the number of AIF and ASWO nodes added during the annotation process.

\subsection{Results and Analysis}

Firstly, we present how the argumentation structure changes and grows over time, in both a logical and rhetorical capacity, by graphing how the number of logical support and attack nodes (i.e. RAand CA-nodes) and rhetorical support and attack nodes (i.e. PSand PC-nodes) changes with each post contributed to the argument 2 . Figures $1 \mathrm{~b}$ and $1 \mathrm{c}$ show that in the cases of Facebook and Reddit use of rhetorical tactics rises slowly compared to the use of logical tactics. However, Figure 1a shows that in the case of Twitter, the rhetorical contributions rise in parallel to the logical contributions. In both samples from Twitter and Reddit, the distribution of logical supports and attacks also remain approximately equal. In all three examples, rhetorical conflict far outweighs rhetorical support. Overall, it appears that there is no sudden shift in tactics from arguing logically to adopting a rhetorical approach - rhetorical argument forms an underlying and consistent strategy throughout the argument $_{2}$.

In addition, we examined the proportion of logical versus rhetorical contributions made by each user. Figures $2 \mathrm{a}$ and $2 \mathrm{c}$ show that users in the Twitter and Reddit cases made more individual contributions to the argumentation structure than those in the Facebook sample, shown in Figure $2 b$. This suggests that there is more engagement in these cases than in the Facebook sample. All samples also display a tendency for rhetorical contributions to be distributed across the scale, with grouping towards either end. This has two connotations; firstly, users that contribute most to a discussion are also most likely to use rhetorical techniques, and secondly, users that contribute no logical contributions at all are most likely to provide a rhetorical contribution.

Correlations were drawn between the structure of the annotated argument graph, including elements such as the number of logical or rhetorical supports or conflicts and replies to and from each post, and features of the post content and structure, such as post length, number of expletives, percentage of spelling errors and again, replies to and from the post. Due to the largely discrete (and often binary) nature of the features and values studied the correlations seen are relatively weak. However, there are some stronger correlations which show potential early indicators of the structure and value of an argument. For example, as might be expected, longer posts are more likely to contain more I-nodes. Posts that use a large number of expletives are likewise more likely to contain a rhetorical attack. When examining all three case studies together, posts made in reply correlated with posts that were replied to, implying that when one or more users engage in a discussion, they are more likely to be engaged with in return. 


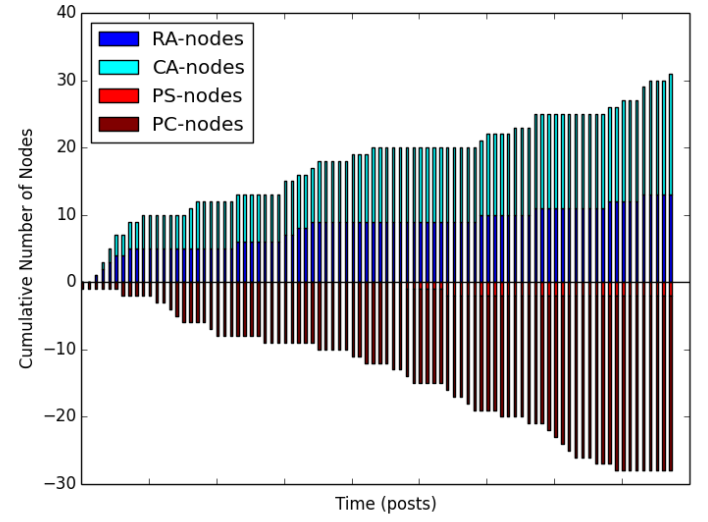

(a) Logical and rhetoric tactics over time on Twitter

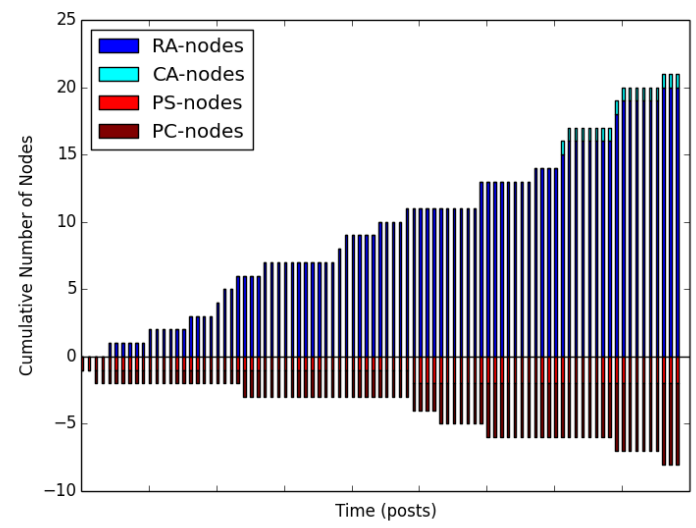

(b) Logical and rhetoric tactics over time on Facebook

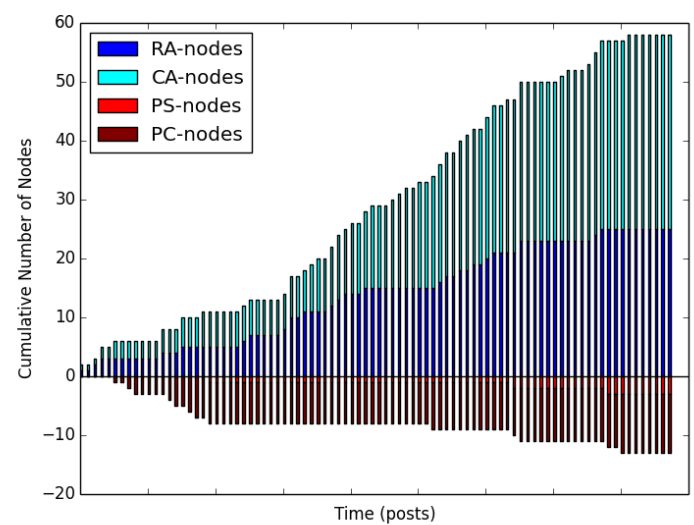

(c) Logical and rhetoric tactics over time on Reddit

Figure 1: Cumulative use of logical and rhetoric tactics over time

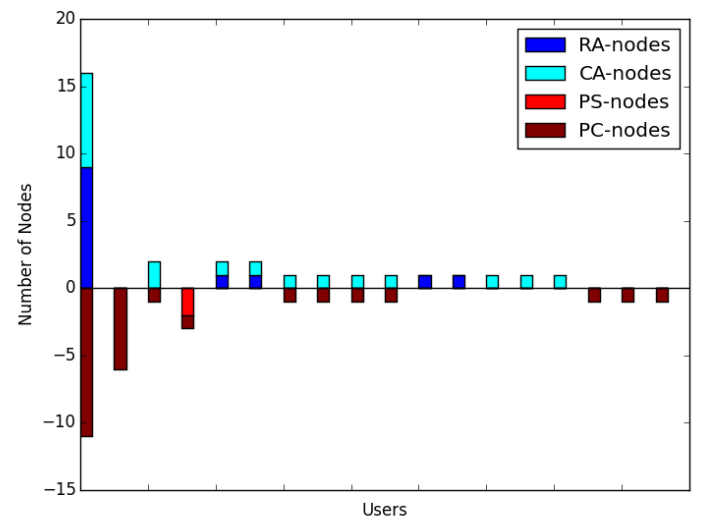

(a) Logical and rhetorical contributions by Twitter users

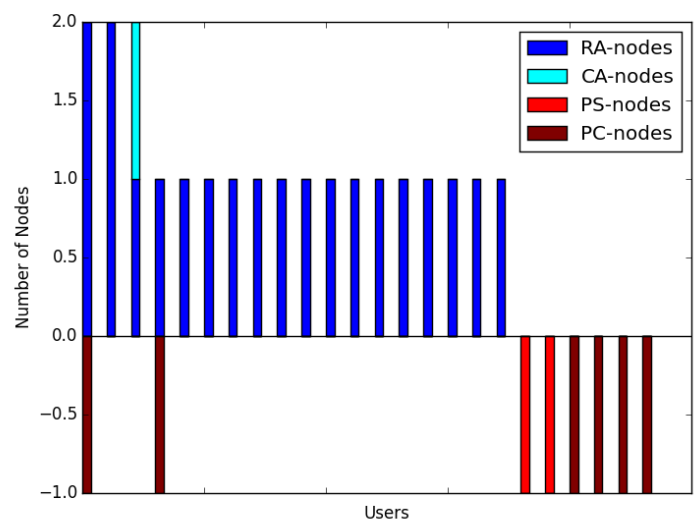

(b) Logical and rhetorical contributions by Facebook users

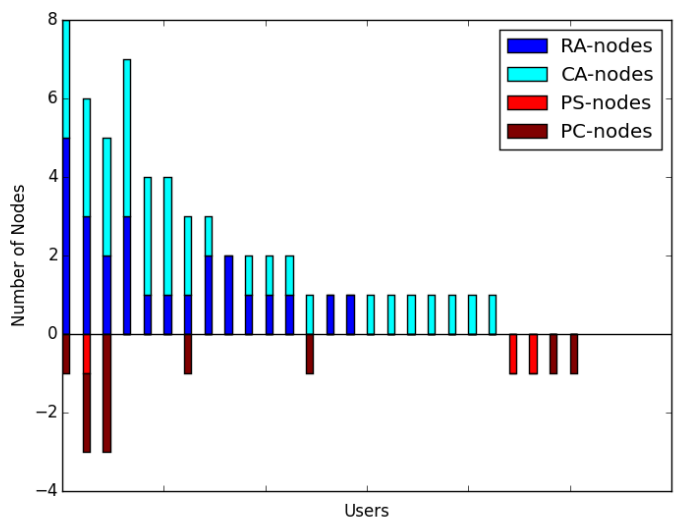

(c) Logical and rhetorical contributions by Reddit users

Figure 2: Logical and rhetorical contributions per sampled user 


\section{CONCLUSIONS AND FUTURE WORK}

Argumentation, like the social web itself, is a diverse construct that is challenging to model but has huge potential if correctly harnessed. To do this, both the logical and rhetorical features must be taken into account, particularly when modelling eristic arguments. The work presented in this paper provides a novel framework for modelling a subset of rhetorical argumentation, ideal for use in modelling social argumentation, then demonstrates some of the structures this allows us to observe when applied to three case studies. From these case studies, we draw three major conclusions. Firstly, and most importantly, rhetorical tactics are shown to be present throughout the argumentation in the case studies, even when only accounting for a small subset of rhetorical argumentation. Clearly, failure to accurately model these social argumentation strategies is detrimental to the goal of studying how discussions evolve on the social web. Secondly, in our three case studies, rhetorical tactics are most often used by either those contributing the most to the discussion overall, or by those who do not contribute logically at all. Finally, while the features of the argumentation structure above are challenging to detect automatically and expensive to manually annotate, the markers present in the social media sphere are relatively trivial to detect. When given enough data, it is possible to draw correlations between these argumentation and social features to give an estimation of the likelihood that a contribution is logical, positively rhetorical or negatively rhetorical. Given enough data, it may also be possible to estimate the weight or impact a given post will have on the overall argumentation structure. However, it must be noted that without further augmentations to the model, the structure of the annotated graph itself gives no indication to the quality of argument present.

There are a number of avenues that can be taken to further this research. Firstly more data can be collected and annotated from the social web to refine the estimates presented here. This can be approached with respect to breadth, by examining additional sites not covered here, such as virtual worlds; or depth, examining multiple additional sites for each biome discussed here, to determine whether the correlations described hold true for each category, or are site-dependant. Secondly, additional annotations can be made with respect to the given case studies. This can also be approached from the perspective of breadth or depth; either categorisation of additional logical and rhetorical strategies or by sub-categorisation of those areas that are currently annotated. Thirdly, a node structure could be applied to the notion of audience perception, to directly reflect the social attributes of the argument 2 as a part of the argumentation structure itself. The computational modelling of social media argumentation has the potential to be a powerful tool in both our understanding of social media use and the development of new tools to encourage more sophisticated argument and counter antisocial behaviour. Current formal models of argument do not well suit the eristic arguments found on the social web, or cope well with the rhetorical tactics used. Our hope is that our work shows both how formal models can be extended to describe these features, and that those descriptions are necessary to create a complete picture of online argumentation.

\section{REFERENCES}

[1] T. Blount, D. E. Millard, and M. J. Weal. Towards Modelling Dialectic and Eristic Argumentation on the Social Web. In 14th workshop on Computational Models of Natural Argument, 2014.

[2] J. G. Breslin, S. Decker, A. Harth, and U. Bojars. SIOC: an approach to connect web-based communities. International
Journal of Web Based Communities, 2(2):133-142, 2006.

[3] C. Chesñevar, J. McGinnis, S. Modgil, I. Rahwan, C. Reed, G. Simari, M. South, G. Vreeswijk, and S. Willmott. Towards an argument interchange format. Knowledge Engineering Review, 21(4):293-316, 2006.

[4] E. Gilbert, T. Bergstrom, and K. Karahalios. Blogs are echo chambers: Blogs are echo chambers. In 42nd Hawaii International Conference on System Sciences, HICSS'09, pages 1-10. IEEE, 2009.

[5] U. Hahn, M. Oaksford, and A. Corner. Circular arguments, begging the question and the formalization of argument strength. In Proceedings of AMKLC'05, International Symposium on Adaptive Models of Knowledge, Language and Cognition, pages 34-40, 2005.

[6] E. A. Jane. "Your a Ugly, Whorish, Slut" Understanding E-bile. Feminist Media Studies, 14(4):531-546, 2014.

[7] C. Jørgensen. Public Debate - An Act of Hostility? Argumentation, 12(4):431-443, 1998.

[8] A. M. Kaplan and M. Haenlein. Users of the world, unite! The challenges and opportunities of Social Media. Business horizons, 53(1):59-68, 2010.

[9] W. Kunz and H. W. Rittel. Issues as elements of information systems, volume 131. Institute of Urban and Regional Development, University of California Berkeley, California, 1970.

[10] C. Lange, U. Bojars, T. Groza, J. G. Breslin, and S. Handschuh. Expressing Argumentative Discussions in Social Media Sites. In Proceedings of the Workshop on Social Data on the Web, Karlsruhe, Germany, 2008.

[11] J. Leskovec and C. Faloutsos. Sampling from large graphs. In Proceedings of the 12th ACM SIGKDD international conference on Knowledge discovery and data mining, pages 631-636. ACM, 2006.

[12] A. d. Moor and M. Aakhus. Argumentation support: From technologies to tools. Communications of the ACM, 49(3):93-98, Mar. 2006.

[13] D. J. O'Keefe. Readings in argumentation, volume 11, chapter 5, pages 79-91. Walter de Gruyter, 1992.

[14] Plato. Book V. The Republic. Basic Books, 380BC. (Bloom, A.D. Trans. 1991).

[15] I. Rahwan, F. Zablith, and C. Reed. Laying the foundations for a world wide argument web. Artificial intelligence, 171(10):897-921, 2007.

[16] C. Reed, S. Wells, J. Devereux, and G. Rowe. AIF+: Dialogue in the Argument Interchange Format. FRONTIERS IN ARTIFICIAL INTELLIGENCE AND APPLICATIONS, 172:311, 2008

[17] J. Schneider, B. Davis, and A. Wyner. Dimensions of argumentation in social media. Lecture Notes in Computer Science, 7603:21-25, 2012.

[18] J. Schneider, S. Villata, and E. Cabrio. Why did they post that argument? Communicative Intentions of Web 2.0 Arguments. In Arguing on the Web 2.0, Amsterdam, 2014. SINTELNET, European Network for Social Intelligence.

[19] W. Sherchan, S. Nepal, and C. Paris. A survey of trust in social networks. ACM Computing Surveys (CSUR), 45(4):47, 2013.

[20] N. E. Willard. Cyberbullying and cyberthreats: Responding to the challenge of online social aggression, threats, and distress. Research Press, 2007. 